\title{
Correlation-Induced Double-Plasmon Excitation in Simple Metals Studied by Inelastic X-Ray Scattering
}

\author{
C. Sternemann, ${ }^{1}$ S. Huotari, ${ }^{2}$ G. Vankó, ${ }^{2}$ M. Volmer, ${ }^{1}$ G. Monaco, ${ }^{2}$ A. Gusarov, ${ }^{3,4}$ H. Lustfeld, ${ }^{3}$ \\ K. Sturm, ${ }^{3}$ and W. Schülke ${ }^{1}$ \\ ${ }^{1}$ Institute of Physics, University of Dortmund, D-44221 Dortmund, Germany \\ ${ }^{2}$ European Synchrotron Radiation Facility, BP 220, F-38043 Grenoble Cedex, France \\ ${ }^{3}$ Institut für Festkörperforschung, Forschungszentrum Jülich GmbH, D-52425 Jülich, Germany \\ ${ }^{4}$ SCK $\cdot$ CEN, Belgian Nuclear Research Centre, Boeretang 200, B-2400 Mol, Belgium
}

(Received 14 April 2005; published 3 October 2005)

\begin{abstract}
We report a new type of peaklike structure observed in the tail of the dynamic structure factor of simple metals, measured by inelastic x-ray scattering. Based on the momentum-transfer dependence of the energy position and the intensity of this structure, it has been unambiguously attributed to intrinsic plasmonplasmon excitations, an electronic correlation effect that was theoretically predicted by many-body perturbation theory of the homogeneous-electron-gas model beyond the random-phase approximation. This signature appears to be largely unaffected by electron-ion interaction effects. Thus a structure that is primarily caused by correlation effects in the electron gas has been found experimentally in the dynamic structure factor of simple metals.
\end{abstract}

DOI: 10.1103/PhysRevLett.95.157401

PACS numbers: 78.70.Ck, 71.10.Ca, 71.45.-d

The appropriate theoretical treatment of correlation in an interacting electron gas at metallic densities still remains a challenge in spite of the large amount of work on this topic. Therefore, experimental methods that can directly test theoretical predictions are very valuable. For systems that closely resemble a homogeneous electron gas (jellium), measurements of the dynamic structure factor $S(\mathbf{q}, \omega)$ as a function of the momentum transfer $\mathbf{q}$ and the energy loss $\omega$ offer such a testing ground of correlation effects, since $S(\mathbf{q}, \omega)$ is the Fourier transform in space and time of the density-density correlation function [1]. Inelastic x-ray scattering (IXS) is the favorable experimental method to study $S(\mathbf{q}, \omega)$ for large momentum transfers q [2], i.e., when short-range correlations are probed. In particular, the advantage of IXS is the nearly complete absence of multiple scattering in contrast to electron energy-loss spectroscopy (EELS) [3], because in EELS extrinsic multiple excitations can obscure the information on electron correlations. IXS studies on simple metals [4] clearly showed significant deviations of the overall shape of $S(\mathbf{q}, \omega)$ for large $\mathbf{q}$ from the shape predicted by the jellium model in the random-phase approximation (RPA) [5]. The RPA is known to incorporate long-range correlations only and thus for small $q=|\mathbf{q}|$ approximately accounts for the energy position and dispersion of collective excitations such as plasmons in simple metals. In both cases the influence of the effective electron-ion interaction is found to be small. For larger $q$ the deviations of the experimental observations from the RPA jellium calculations exhibit three characteristic features: (i) the centroid of $S(\mathbf{q}, \omega)$ as a function of $\omega$ is shifted to lower energy losses [4]; (ii) a double-peak or a one-peak-one-shoulder structure appears to be universal for all simple metals [5]; (iii) the $S(\mathbf{q}, \omega)$ spectra show tails for $\omega$ far beyond the upper limit of the jellium (single) particle-hole excitation spectrum [6,7].

All three observed features were attributed to correlation effects beyond the RPA [4,8-13]. However, the ion potential was found to cause similar effects [13-18]. As a result, it is difficult to unambiguously identify specific correlation effects from these experiments.

In search of a unique manifestation of correlation effects, one notes that as mentioned above the plasmon is a unique excitation of an interacting electron gas. In simple metals their observed existence and dispersion is reasonably well described by the jellium model within the RPA. Beyond the RPA, plasmon-plasmon (pl-pl) excitations as a specific correlation effect of the jellium model were also predicted long ago $[19,20]$. These resulted from an analysis of diagrams that are obtained from an expansion of the proper polarizability beyond the RPA to second order in the dynamically RPA-screened Coulomb interaction. The three diagrams (A, B) of first order and the two diagrams (C) of second order together form a consistent set [21] and are depicted in Fig. 1. While the evaluation of the imaginary parts of these diagrams by Hasegawa and Watabe [19] (see also Ref. [20]) is valid only in the limit of small $q$ where the intensity of pl-pl excitations is vanishingly small, the extension of the calculation to arbitrary $q$ and $\omega$ outside the (single) particle-hole excitation spectrum was accomplished only recently [13]. With increasing $q$ this correlation effect becomes large enough to be observed experimentally as is reported in this Letter.

The dynamic structure factor can be expressed in terms of the dielectric function $\epsilon(\mathbf{q}, \omega)$, which is related to the proper polarizability $\pi_{\mathrm{p}}(\mathbf{q}, \omega)$ by

$$
\epsilon(\mathbf{q}, \omega)=1+v(q) \pi_{\mathrm{p}}(\mathbf{q}, \omega),
$$



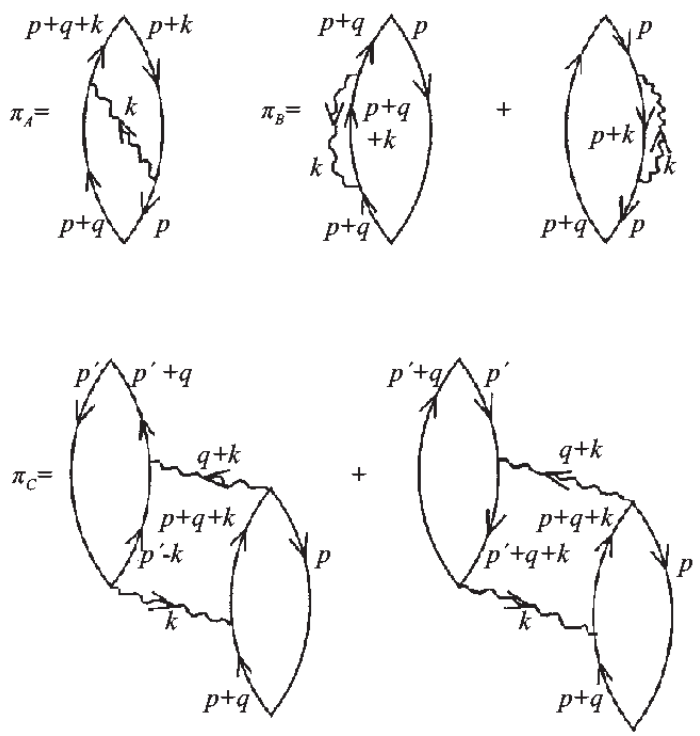

FIG. 1. A, B, and C diagrams, lowest order corrections to the RPA bubble. The wavy lines denote the dynamically RPAscreened Coulomb interaction $\left(p, p^{\prime}, q, k\right.$ are four-dimensional vectors including frequencies).

with $v(q)=4 \pi / q^{2}$. Atomic units (a.u.) are used with $\hbar=$ $m=e=1$. For the jellium model, $\pi_{\mathrm{p}}(\mathbf{q}, \omega)$ depends on $q=|\mathbf{q}|$ only. In the approximation considered here, we have

$$
\begin{aligned}
\pi_{\mathrm{p}}(\mathbf{q}, \omega)= & \pi_{0}(\mathbf{q}, \omega)+\pi_{\mathrm{A}}(\mathbf{q}, \omega)+\pi_{\mathrm{B}}(\mathbf{q}, \omega)+\pi_{\mathrm{C}}(\mathbf{q}, \omega) \\
& +\cdots
\end{aligned}
$$

where the RPA bubble $\pi_{0}(\mathbf{q}, \omega)$ defines the well known Lindhard dielectric function $\epsilon_{\mathrm{L}}(\mathbf{q}, \omega)=1+$ $v(q) \pi_{0}(\mathbf{q}, \omega)$, and $\pi_{\mathrm{A}}(\mathbf{q}, \omega)+\pi_{\mathrm{B}}(\mathbf{q}, \omega)+\pi_{\mathrm{C}}(\mathbf{q}, \omega)$ is the sum of the expressions corresponding to the diagrams depicted in Fig. 1. They define correlation effects as the leading corrections to the RPA. It was demonstrated that a consistent set of diagrams must include the $\pi_{\mathrm{C}}(\mathbf{q}, \omega)$ diagrams [21]. Outside the particle-hole excitation spectrum $\epsilon_{\mathrm{L}}(\mathbf{q}, \omega)$ is real and we find for the dynamic structure factor

$$
\begin{array}{r}
S(\mathbf{q}, \omega)=\frac{q^{2}}{4 \pi^{2} n_{0}} \operatorname{Im}\left(\frac{-1}{\epsilon(\mathbf{q}, \omega)}\right) \approx \frac{1}{\pi n_{0}} \\
\times \frac{\operatorname{Im}\left[\pi_{0}(\mathbf{q}, \omega)+\pi_{\mathrm{A}}(\mathbf{q}, \omega)+\pi_{\mathrm{B}}(\mathbf{q}, \omega)+\pi_{\mathrm{C}}(\mathbf{q}, \omega)\right]}{\epsilon_{\mathrm{L}}^{2}(\mathbf{q}, \omega)},
\end{array}
$$

where $n_{0}$ is the electron density. The pl-pl excitations result from the evaluation of the $\mathrm{C}$ diagrams of Fig. 1, because the dynamically RPA-screened Coulomb potential enters in second order; i.e., RPA screening yields the product $1 / \epsilon_{\mathrm{L}}\left(\mathbf{q}+\mathbf{k}, \omega+\omega^{\prime}\right) \cdot 1 / \epsilon_{\mathrm{L}}\left(\mathbf{k}, \omega^{\prime}\right)$. Here $\mathbf{k}$ and $\omega^{\prime}$ are internal momentum and energy variables to be integrated over. The poles of the two involved screening functions give rise to the excitation of two plasmons at an energy $\omega=\omega_{\mathrm{p}}(k)+\omega_{\mathrm{p}}(|\mathbf{q}+\mathbf{k}|)$. Since plasmons can exist only for $k \leq q_{\mathrm{c}}$ and $|\mathbf{q}+\mathbf{k}| \leq q_{\mathrm{c}}$, where $q_{\mathrm{c}}$ is the plasmon cutoff wave number, the pl-pl excitations can occur only for $0 \leq q \leq 2 q_{\mathrm{c}}$. For fixed $q$ the onset of the two-plasmon structure in the tail of $S(\mathbf{q}, \omega)$ is given by $\omega_{\mathrm{pl}-\mathrm{pl}}^{\text {onset }}(q)=$ $2 \omega_{\mathrm{p}}(q / 2)=2 \omega_{\mathrm{p}}(0)+\alpha q^{2} / 2$ with the free electron plasmon frequency $\omega_{\mathrm{p}}=\omega_{\mathrm{p}}(0)+\alpha q^{2}$ and the plasmon dispersion constant $\alpha$. The upper $\omega$ limit for pl-pl excitations is given by $\omega_{\mathrm{pl}-\mathrm{pl}}^{\lim }(q)=2 \omega_{\mathrm{p}}\left(q_{\mathrm{c}}\right)=2\left(q_{\mathrm{c}}^{2} / 2+k_{\mathrm{F}} q_{\mathrm{c}}\right)$, with the Fermi momentum $k_{\mathrm{F}}$. Thus the expected structure for pl-pl excitation has a rather limited extension on the energy-loss scale. Therefore, the peaklike pl-pl excitation structure can easily be distinguished from the lessstructured contribution of the particle-hole-pair-particlehole-pair excitations and the particle-hole-pair-plasmon excitations. It must be mentioned that the prerequisites of the calculations of Ref. [13] break down when the free (single) particle-hole continuum approaches the pl-pl feature. The energy-loss position of the maximum of the pl-pl excitation [13] is given by

$$
\omega_{\mathrm{pl}-\mathrm{pl}}^{\max }(q) \approx 2 \omega_{\mathrm{p}}(0)+\alpha q^{2} .
$$

The existence of the pl-pl excitations was verified experimentally by observing the corresponding fingerprints in $S(\mathbf{q}, \omega)$ of Al single crystals with $\mathbf{q} \|[100]$ and [110]. The dynamic structure factor was measured by means of IXS with high statistical accuracy. Peaklike structures were found and, after a comparison with corresponding theoretical predictions, attributed to pl-pl excitations based on the $q$ dependence of both their peak position and intensity. The pl-pl contributions of both diagrams of second order in the dynamically screened Coulomb interaction (diagrams C) were computed for $\mathrm{Al}\left(r_{\mathrm{s}}=2.07, r_{\mathrm{s}}\right.$ is the free electron gas parameter) and $\mathrm{Na}\left(r_{\mathrm{s}}=3.93\right)$ for the $q$ values of the experiment based on Ref. [13].

The experiments were carried out at the beam line ID16 of the European Synchrotron Radiation Facility (Grenoble, France) utilizing a Rowland-type spectrometer with a $1 \mathrm{~m}$ diameter Rowland circle and a spherically bent $\mathrm{Si}(555)$ analyzer crystal. The analyzer energy was kept fixed at $9.89 \mathrm{keV}$ and the incident-photon energy was tuned across the required energy-loss range of $75 \mathrm{eV}$. The overall energy resolution was estimated from the width of the quasielastic line to be $1.5 \mathrm{eV}$. The momentum-transfer resolution was 0.1 a.u. The dynamic structure factor was measured on $\mathrm{Al}$ single crystals $\left(q_{\mathrm{c}}=0.73\right.$ a.u. $)$ for $q / q_{\mathrm{c}}$ between 0.73 and 1.21 with q $\|[100]$ and [110]. Several single spectra were measured to obtain the full energy-loss spectrum up to the Al $L$ edge, normalized to the incident-beam intensity and summed up. Finally, the spectra were converted into the dynamic structure factor by using the $f$-sum rule [6]. Between 27 and $50 \mathrm{eV}$ the data were measured with higher statistical accuracy.

The experimental results of $S(\mathbf{q}, \omega)$ for single crystalline Al with q $\|[100]$ are presented in Fig. 2 on a semilogarithmic scale for all measured $q$ values as indicated. The pl$\mathrm{pl}$ peak is marked by the arrow. It is clearly visible in the high energy-loss tail of $S(\mathbf{q}, \omega)$ and disperses in its energy- 
loss position with increasing $q$. For $q \geq q_{\mathrm{c}}$ the pl-pl peak vanishes slowly into the particle-hole continuum. The contribution of the double-plasmon excitation to the dynamic structure factor has been extracted by subtraction of its continuously decreasing tail. The tail was obtained by fitting to the shape of $S(\mathbf{q}, \omega)$ a weighted sum of a Pearson VII function [22], an exponential and a small background second order in $\omega$ at both sides of the pl-pl feature. As an example, the result of this fit for $q / q_{\mathrm{c}}=$ 1.01 is shown in Fig. 2 as a solid line. Since the particlehole contribution extends to one side of the pl-pl feature with increasing $q$, the fit function provides a proper description of the tail only for $q / q_{\mathrm{c}}<1.21$. The $\mathrm{pl}$-pl features obtained by using this subtraction procedure are presented in Fig. 3(a), while in Fig. 3(b) the computed spectra are shown, which have been convoluted by the experimental energy resolution function. The error bars indicate the statistical accuracy of the measurements due to the count rate.

Within the limits of statistical accuracy no significant $\mathbf{q}$-orientation dependence of the pl-pl peak could be found even though the shape of $S(\mathbf{q}, \omega)$ is strongly q-orientation dependent. The agreement between the experimental and the theoretical results both with respect to the dispersion of the energy position and the $q$ dependence of the intensity evolution is remarkable. However, the absolute experimental intensities are approximately 50\% smaller than the theoretical ones, which will be commented on later. The position of the pl-pl peak on the energy-loss scale has been estimated as a function of $q$ for experimental and theoretical spectra presented in Figs. 3(a) and 3(b). The dispersion relation for $\omega_{\mathrm{pl}-\mathrm{pl}}^{\max }(q)$ was fitted to the corresponding data sets by varying $\alpha$ and $\omega_{\mathrm{p}}(0)$. The fit to the experimental results yields $\alpha=0.27 \pm 0.02$ and $\omega_{\mathrm{p}}^{\mathrm{e}}(0)=15.2 \pm$ $0.2 \mathrm{eV}(15.1 \pm 0.2 \mathrm{eV})$ for $\mathbf{q} \|[100]([110])$. For the theo-

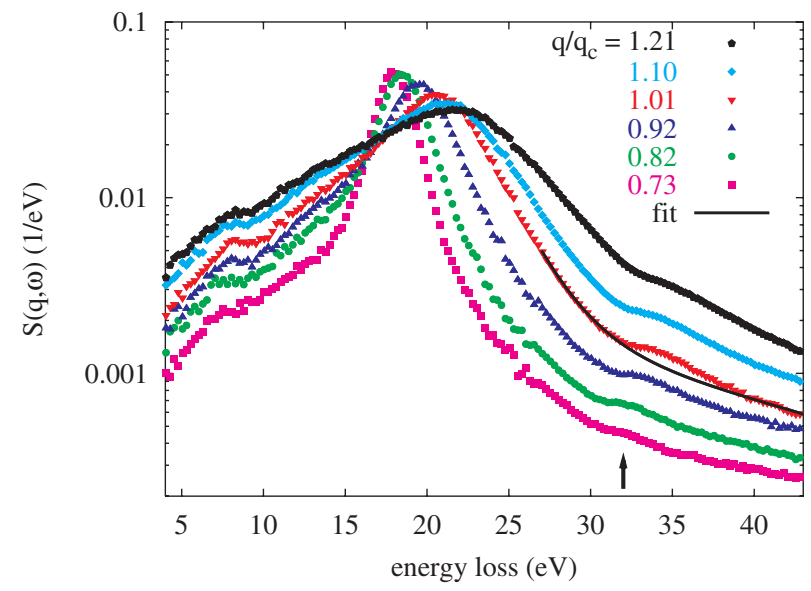

FIG. 2 (color online). Experimental data of the dynamic structure factor of $\mathrm{Al}$ for different $q / q_{\mathrm{c}}$ as indicated with momentum transfer $\mathbf{q} \|[100]$ on an absolute logarithmic scale. The arrow indicates the pl-pl structure and the solid line represents a fit to the $S(\mathbf{q}, \omega)$ tail for $q / q_{\mathrm{c}}=1.01$ (see text for details). retical pl-pl peak $\alpha=0.27$ and $\omega_{\mathrm{p}}^{\mathrm{t}}(0)=16.2 \mathrm{eV}$ have been obtained. The dispersion constants $\alpha$ of experiment and theory are in good agreement. The average difference $\Delta \omega_{\mathrm{pl}-\mathrm{pl}}(0)=1.05 \pm 0.2 \mathrm{eV}$ between $\omega_{\mathrm{p}}^{\mathrm{t}}(0)$ and $\omega_{\mathrm{p}}^{\mathrm{e}}(0)$ is most probably due to the ion-electron interaction and it is somewhat larger than $\Delta \omega_{\mathrm{p}}(0)=0.8 \mathrm{eV}$, the difference between $\omega_{\mathrm{p}}^{\mathrm{RPA}}(0)=15.8 \mathrm{eV}$ and $\omega_{\mathrm{p}}^{\mathrm{EELS}}(0)=15.0 \mathrm{eV}$ [23]. This might be due to the fact that the description of the plasmon dispersion of crystalline matter by a single dispersion constant as done here is obviously a simplification. The agreement between $\omega_{\mathrm{p}}^{\mathrm{e}}(0)$ and $\omega_{\mathrm{p}}^{\mathrm{EELS}}(0)$ is very satisfying. Furthermore, the energy-loss-integrated intensities of the pl-pl features have been estimated as a function of $q$. A $q^{5.3}$ dependency was obtained for theory from a fit to the calculated data. The experiment follows this $q$ dependence satisfactorily, of course with by $50 \%$ smaller absolute values.

Measurements of the pl-pl-excitation features in $S(\mathbf{q}, \omega)$ on polycrystalline $\mathrm{Na}[24]$ also exhibit rather good agreement with calculation as far as peak dispersion is con-
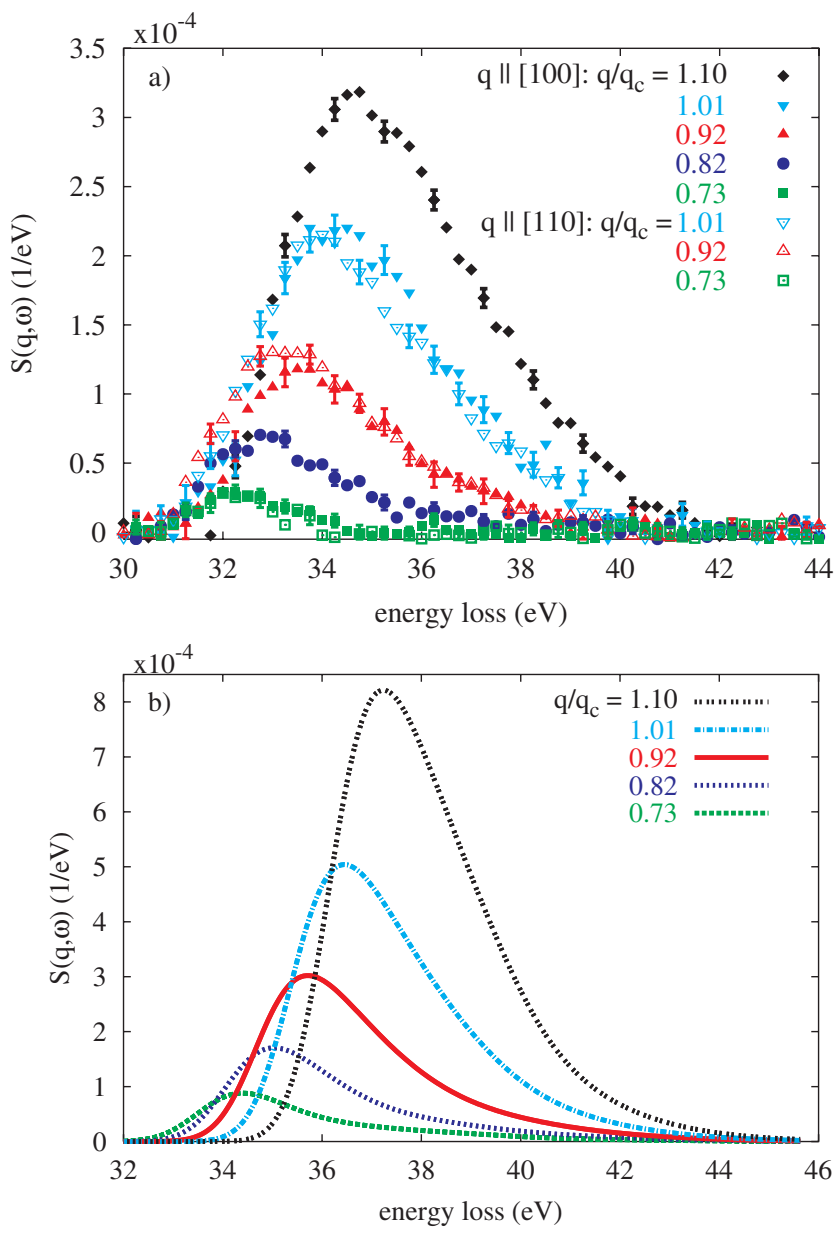

FIG. 3 (color online). (a) Al pl-pl correlation peak extracted from the experimental $S(\mathbf{q}, \omega)$ spectra as described within the text. (b) Calculated Al pl-pl correlation peak spectra for $r_{\mathrm{s}}=$ 2.07 on an absolute scale, broadened by the experimental resolution. 
cerned. The fit of the experimental results to $\omega_{\mathrm{pl}-\mathrm{pl}}^{\max }(q)$ yields a value of $\omega_{\mathrm{p}}^{\mathrm{e}}(0)=5.6 \pm 0.2 \mathrm{eV}$, which is found to be in agreement with $\omega_{\mathrm{p}}^{\mathrm{EELS}}(0)=5.77 \mathrm{eV}$ [25]. Furthermore, the dispersion constant $\alpha=0.21 \pm 0.02$ is in line with the corresponding theoretical value of $\alpha=$ 0.22 obtained from the results of the calculations performed for the Na pl-pl excitation. As far as the integrated intensity of the Na pl-pl structure and its $q$ dependence is concerned theory and experiment show reasonable agreement. The magnitude of the experimental intensity of the pl-pl feature of $\mathrm{Na}$ is clearly larger than that of $\mathrm{Al}$ when considered for the same values of $q / q_{\mathrm{c}}$ which is in qualitative agreement with theory [13].

It is important to point out that Monte Carlo simulations performed to study the effect of multiple scattering in the measurement of $S(\mathbf{q}, \omega)$ of simple metals [26] clearly show that the distinct peaklike tail structure of $S(\mathbf{q}, \omega)$, which we have attributed to a correlation-induced intrinsic pl-pl excitation, cannot result from extrinsic multiple losses. In fact, these calculations for $\mathrm{Al}$ have shown that in the range of $\omega=2 \omega_{\mathrm{p}}$ the doubly scattered contribution to $S(\mathbf{q}, \omega)$ was not larger than $0.2 \%$ of the peak value and depends only weakly on $q$. In contrast, the peak contribution of the intrinsic pl-pl excitations exceeds $1 \%$ of the $S(\mathbf{q}, \omega)$ peak value for $q \approx q_{\mathrm{c}}$ and is strongly $q$ dependent.

In conclusion, new structures have been found in the experimental high energy tail of $S(\mathbf{q}, \omega)$, as measured by inelastic $\mathrm{x}$-ray scattering on single crystal $\mathrm{Al}$ and polycrystalline Na. These structures can clearly be attributed to intrinsic double-plasmon excitations as a genuine correlation effect and are not caused by the weak effective crystal potential. We found remarkable agreement between theory and experiment for $\mathrm{Al}$ with respect to (i) the spectral position of the pl-pl excitations, (ii) the dispersion of the maxima of the pl-pl spectra, as well as (iii) the $q$ dependence of the integrated intensities. However, there is a discrepancy between theory and experiment concerning the absolute intensities, which can be due to the so-called exchange counterparts of the $\mathrm{C}$ diagrams shown in Fig. 1, not taken into account here $[27,28]$. So far the imaginary parts of the exchange diagrams could be evaluated only in the limit of high energies, i.e., $\omega \gg q^{2} / 2+q k_{\mathrm{F}}$, when (dynamical) screening is unimportant [27] and hence far above the energy regime of the double-plasmon excitations. There they reduce the contribution from the diagrams of Fig. 1 by $50 \%$. On the other hand, as pointed out in Ref. [21], this set of exchange diagrams is associated with the bare second order exchange energy $\epsilon_{\mathrm{ex}, 2}$ which is independent of $r_{\mathrm{s}}$. From the ground-state-energy theorem [1] we infer that their contribution to $S(\mathbf{q}, \omega)$ should not increase with increasing $r_{\mathrm{s}}$ so that their relative importance could be larger for $\mathrm{Al}$ than for $\mathrm{Na}$. The evaluation of the exchange diagrams are left as a challenge to theory. Nevertheless, the good agreement of the experiment with the theoretical predictions from the jellium model gives confidence in the diagrammatic expansion of the proper polarizability when dealing with dynamical exchange and correlation effects in systems that closely resemble the jellium model.

C. S. kindly acknowledges the support and encouragement from M. Tolan. The authors would like to thank C. Henriquet for his technical assistance and are grateful to $\mathrm{H}$. Müller for expert advice in preparing the Na samples. We acknowledge the European Synchrotron Radiation Facility for provision of synchrotron radiation.

[1] D. Pines, Elementary Excitations in Solids (Benjamin, New York, 1964).

[2] W. Schülke, J. Phys. Condens. Matter 13, 7557 (2001).

[3] H. Raether, Excitation of Plasmons and Interband Transitions by Electrons (Springer, Berlin, 1980).

[4] P. M. Platzman and P. Eisenberger, Solid State Commun. 14, 1 (1974).

[5] P. M. Platzman and P. Eisenberger, Phys. Rev. Lett. 33, 152 (1974).

[6] W. Schülke et al., Phys. Rev. B 40, 12215 (1989).

[7] W. Schülke et al., Phys. Rev. B 52, 11721 (1995).

[8] S. Rahman and G. Vignale, Phys. Rev. B 30, 6951 (1984).

[9] F. Green et al., Phys. Rev. B 31, 2796 (1985).

[10] P. M. Platzman et al., Phys. Rev. B 46, 12943 (1992).

[11] L. X. Benedict and E. L. Shirley, Phys. Rev. B 59, 5441 (1999).

[12] C. Sternemann et al., Phys. Rev. B 62, R7687 (2000).

[13] K. Sturm and A. Gusarov, Phys. Rev. B 62, 16474 (2000).

[14] W. Schülke, H. Schulte-Schrepping, and J. R. Schmitz, Phys. Rev. B 47, 12426 (1993).

[15] N. E. Maddocks, R. W. Godby, and R. J. Needs, Europhys. Lett. 27, 681 (1994).

[16] N.E. Maddocks, R. W. Godby, and R. J. Needs, Phys. Rev. B 49, 8502 (1994).

[17] A. Fleszar, A. A. Quong, and A. G. Eguiluz, Phys. Rev. Lett. 74, 590 (1995).

[18] C. Sternemann, A. Kaprolat, and W. Schülke, Phys. Rev. B 57, 622 (1998).

[19] M. Hasegawa and M. Watabe, J. Phys. Soc. Jpn. 27, 1393 (1969).

[20] D.F. DuBois and M. G. Kivelson, Phys. Rev. 186, 409 (1969).

[21] D. J. W. Geldart and S. H. Vosko, Can. J. Phys. 44, 2137 (1966).

[22] P. S. Prevéy, Adv. X-Ray Anal. 29, 103 (1986).

[23] J. Sprösser-Prou, A. vom Felde, and J. Fink, Phys. Rev. B 40, 5799 (1989).

[24] S. Huotari et al. (to be published).

[25] A. vom Felde, J. Sprösser-Prou, and J. Fink, Phys. Rev. B 40, 10181 (1989).

[26] J. Felsteiner and W. Schülke, Nucl. Instrum. Methods Phys. Res., Sect. B 132, 1 (1997).

[27] A. J. Glick and W. F. Long, Phys. Rev. B 4, 3455 (1971).

[28] M.E. Bachlechner, A. Holas, H. M. Böhm, and A. Schinner, Phys. Rev. B 54, 2360 (1996). 